\title{
Fotossensibilização em ovinos associada à ingestão de Brachiaria brizantha no estado do Pará1
}

\author{
Tatiane Teles Albernaz ${ }^{2^{*}}$, José Alcides Sarmento da Silveira², Natália \\ da Silva e Silva², Cairo Henrique Sousa Oliveira², Alessandra dos \\ Santos Belo Reis ${ }^{2}$, Carlos Magno Chaves Oliveira², Marcos Dutra \\ Duarte $^{2}$ e José Diomedes Barbosa ${ }^{2}$
}

\begin{abstract}
Albernaz T.T., Silveira J.A.S., Silva N.S., Oliveira C.H.S., Belo Reis A.S., Oliveira C.M.C., Duarte M.D. \& Barbosa J.D. 2010. [Photosensitization of sheep kept on Brachiaria brizantha pasture in the state of Pará.] Fotossensibilização em ovinos associada à ingestão de Brachiaria brizantha no estado do Pará. Pesquisa Veterinária Brasileira 30(9): 741-748. Central de Diagnóstico Veterinário, Universidade Federal do Pará, Campus de Castanhal, Rua Maximino Porpino da Silva 1000, Pirapora, Castanhal, PA 68743-080, Brazil. E-mail: tatyalbernaz@ufpa.br

Two outbreaks of photosensitization by Brachiaria brizantha were studied and an experiment was performed in Santa Inês and Dorper crossbred sheep, two to three months old, on a farm in Santa Luzia do Pará municipality. These animals were kept from birth until about two months of age in a suspended stall floor, fed purple elephant grass (Pennisetum purpureum cv. purple), and had minerals and water ad libitum. After this period they were placed into a paddock of $B$. brizantha. At the time of the outbreaks and the experimental studies, the farm was visited for epidemiological assessment and clinical examination of the sheep, collection of blood samples for measurement of gamma glutamyltransferase, aspartate aminotransferase, conjugated bilirubin, unconjugated bilirubin, urea, and creatinine. Pasture samples were collected for saponin determination and count of Pithomyces chartarumspores. Necropsies with collection of material for histopathological studies were performed. Outbreak 1 occurred at the time of low rainfall, when the grass was scarce and mature, and morbidity and lethality was $43.4 \%$ and $81.6 \%$, respectively. Outbreak 2 occurred at the beginning of the rainy season, with morbidity and lethality rates of $16.3 \%$ and $76.9 \%$, respectively. From the 50 animals in the experiment, 10 received three times $200 \mathrm{ml}$ of rumen fluid taken from mother sheep of the same batch. The first of these administrations was given one day before the sheep were introduced into the pasture and the other two at weekly intervals. After 15 days in the pasture, the animals were unquiet, looked for shade, had edema of the ears, yellowish mucosae, were apathic, had anorexia and showed sloughing of the skin followed by crusting in some areas of the body. Both, the sheep of the outbreaks as those from the experiment, showed increased levels of GGT, AST, BD, BI, BT, urea and creatinine. In sheep which received ruminal fluid the values of urea and GGT were similar to those which did not receive the ruminal fluid. The creatinine, AST and bilirrubine values were lower in sheep that received the ruminal fluid. Two types of saponins, methylprotodioscin and protodioscin, were detected in the samples of $B$. brizanthafrom the outbreaks and the experiment. The level of saponins in Outbreak 1 and 2 was $0.92 \%$ and $0.88 \%$ respectively. The levels of saponins in the experiment ranged from $1.13 \%$ to $1.62 \%$. The numbers of spores of Pithomyces chartarum in both outbreaks and in the experiment were negligible. At necropsy there was widespread jaundice, the liver was brown-yellow with marked lobular pattern and increased consistency and the kidneys were enlarged yellowish-green. Histological changes occurred mainly
\end{abstract}

1 Recebido em 27 de março de 2010.

Aceito para publicação em 12 de abril de 2010

Parte de Dissertação de Mestrado da primeira autora.

\footnotetext{
${ }^{2}$ Central de Diagnóstico Veterinário (Cedivet), Universidade Federal do Pará (UFPA), Rua Maximino Porpino da Silva 1000, Pirapora, Castanhal, PA 68743-080, Brasil. *Autor para correspondência: tatyalbernaz@ ufpa.br
} 
in the liver and consisted of mild proliferation of bile ducts in portal tracts, presence of binucleate hepatocytes, foamy macrophages, necrosis of isolated hepatocytes, cholangitis, presence of crystals in hepatocytes and macrophages.

INDEX TERMS: Diseases of sheep, photosensitization, Brachiaria brizantha, saponins.

RESUMO-- Foram estudados dois surtos e realizado um experimento de fotossensibilização associada à ingestão por Brachiaria brizantha em ovinos mestiços de Santa Inês e Dorper, com idade variando de dois a três meses, em uma fazenda no município de Santa Luzia do Pará. Esses animais foram mantidos desde o nascimento até aproximadamente dois meses de idade, em apriscos suspensos do chão, recebendo capim-elefante roxo (Pennisetum purpureum cv. roxo), concentrado, sal mineral e água ad libitum. Após esse período foram introduzidos em um piquete de $B$. brizantha. $\mathrm{Na}$ ocasião dos surtos e do experimento a fazenda foi visitada para observação dos dados epidemiológicos, avaliação clínica dos animais, colheita de amostras de sangue para dosagem de GGT, AST, BD, BI, BT, ureia e creatinina e colheita de pastagem para pesquisa de Pithomyces chartarum e saponinas. Também foi realizada necropsia com colheita de material para estudo histológico. O surto 01 ocorreu na época de escassez de chuva, com taxa de morbidade e letalidade de $43,4 \%$ e $81,6 \%$, respectivamente. O surto 02 aconteceu no início da época chuvosa, com taxas de morbidade e letalidade de $16,3 \%$ e $76,9 \%$, respectivamente. Em ambos os surtos o capim encontrava-se com massa residual reduzida e senescente. Dos 50 animais do experimento, 10 receberam $200 \mathrm{ml}$ de fluido ruminal retirado de ovelhas mães do mesmo lote, a primeira administração foi feita um dia antes da introdução desses animais na pastagem, e mais duas subsequentes com intervalo de uma semana. Após 15 dias de pastejo, os animais começaram a apresentar inquietação, procura por sombra, edema nas orelhas, mucosas amareladas, apatia, anorexia e desprendimento da pele seguido por formação de crostas em algumas áreas do corpo. Tanto os animais dos surtos quanto do experimento apresentaram aumento nos níveis de GGT, AST, BD, BI, BT, ureia e creatinina. Os valores de ureia e GGT dos animais que receberam fluido ruminal e dos que não receberam foram semeIhantes, já os valores de creatinina, AST e bilirrubinas foram menores nos animais que receberam fluido ruminal em comparação aos que não receberam. Foram determinados dois tipos de saponinas nas amostras de $B$. brizantha dos surtos e do experimento, a metilprotodioscina e a protodioscina. $O$ nível de saponina no surto 01 e 02 foi $0,92 \%$ e $0,88 \%$, respectivamente. Os níveis de saponinas no experimento variaram de $1,13 \%$ a $1,62 \%$. A quantidade de Pithomyces chartarum, tanto nos surtos quanto no experimento, foi insignificante. Na necropsia foi verificada icterícia generalizada, fígado com consistência aumentada de coloração amarelada e com padrão lobular acentuado. Nos rins foi observada coloração amarelo-esverdeado e aumento de tamanho. As alterações histológicas ocorreram principalmente no fígado e consistiram de leve proliferação das vias biliares nos espaços porta, presença de hepatócitos binucleados, presença de ma- crófagos espumosos, necrose incipiente de hepatócitos isolados, colangite, presença de cristais em macrófagos e hepatócitos.

TERMOS DE INDEXAÇÃO: Doenças de ovinos, fotossensibilização, Brachiaria brizantha, saponinas.

\section{INTRODUÇÃO}

A maioria dos ovinos da região Norte do Brasil é criada em sistema extensivo, em pastagens formadas por Brachiaria spp. Os maiores problemas na utilização das Brachiarias spp. são as cigarrinhas, a degradação e os casos esporádicos de fotossensibilização hepatógena (Resende et al. 2008). A fotossensibilização observada em pastagens de Brachiaria spp. era atribuída à toxina esporodesmina produzida por esporos do fungo Pithomyces chartarum nas pastagens (Döbereiner et al. 1976, Fioravanti 1999). Atualmente, a toxicidade para animais em pastagens de Brachiaria spp. é atribuída à presença de saponinas esteroidais litogênicas na própria gramínea (Smith \& Miles 1993, Lemos et al. 1997). A intoxicação por Brachiaria spp. ocorre em qualquer época do ano, principalmente em bezerros, próximo ao desmame. Ovinos são mais suscetíveis do que bovinos e animais jovens mais do que os adultos. A intoxicação pode ocorrer também em animais lactentes, com menos de trinta dias de idade (Lemos et al. 1996b). Os animais introduzidos pela primeira vez em pastagem de Brachiariaspp. são mais suscetíveis à intoxicação (Lemos et al. 1996b). Para ovinos, as pastagens de Brachiaria spp. são aparentemente menos tóxicas se forem mantidas com pouco centímetros de altura, mediante pastejo intensivo e contínuo (Brum et al. 2004). A fotossensibilização por B. decumbens, B. humidicola e B. brizantha, no Brasil, afeta bovinos (Lemos et al. 1997, Driemeier et al. 2002) e por B. decumbens afeta ovinos (Lemos et al. 1996a, Brum 2006), caprinos (Lemos et al. 1998) e bubalinos (Rozza et al. 2004,). No estado do Pará tem sido diagnosticado fotossensibilização causada pela ingestão de B. humidicola em bubalinos, bovinos (Dados não publicados) e equinos (Barbosa et al. 2006) e pela ingestão de B. brizantha em bovinos (Dados não publicados), ovinos (Albernaz et al. 2008) e caprinos (Silveira et al. 2009). O objetivo deste trabaIho foi estudar dois surtos de fotossensibilização em cordeiros mantidos em pastagem de $B$. brizantha e realizar um experimento com cordeiros no mesmo tipo de pastagem para caracterizar os fatores epidemiológicos, o quadro clínico-patológico e o perfil bioquímico hepático e renal.

\section{MATERIAL E MÉTODOS}

Este estudo foi realizado em uma fazenda localizada no município de Santa Luzia do Pará, mesorregião do nordeste paraense, estado do Pará. Essa mesorregião possui um clima quente e úmido, com temperaturas médias entre $25^{\circ} \mathrm{C}$ e $27^{\circ} \mathrm{C}$ e chuvas que ultrapassam 2000 mm/ano (IBGE 2008). Para o experimen- 
to foram utilizados 49 cordeiros, cruzas Santa Inês x Dorper, com idade variando de 2-3 meses. O experimento foi realizado na mesma fazenda que ocorreram os surtos espontâneos da intoxicação, no período de novembro a dezembro de 2008, época que corresponde a baixos índices pluviométricos na região. Nessas circunstâncias a pastagem se encontrava com massa residual reduzida e senescente. Esses cordeiros foram mantidos, durante 1-2 meses após o nascimento em apriscos suspensos e recebiam capim-elefante roxo (Pennisetum purpureum) cv. roxo, concentrado comercial para ovinos, sal mineral e água ad libitum. Após esse período foram introduzidos em um piquete de $B$. brizantha, recebendo concentrado comercial para ovinos, sal mineral e água ad libitum. Dentre esses 49 animais, 10 receberam $200 \mathrm{ml}$ de fluido ruminal, que havia sido coado em gaze de três faces e administrado através de sonda oroesofágica para pequenos ruminantes; o conteúdo ruminal era retirado de ovelhas mães do mesmo lote, cuja alimentação consistia de capim $B$. brizantha; a primeira administração foi feita um dia antes da introdução desses animais na pastagem, e mais duas subsequentes com intervalo de uma semana. $\mathrm{Na}$ ocasião dos surtos e durante o experimento (um dia antes de serem introduzidos na pastagem e a cada sete dias após a introdução, por um período de sete semanas) a fazenda foi visitada para levantamento dos dados epidemiológicos, observação dos sinais clínicos, colheita de amostras de pastagens, coIheita de amostras de sangue e nos casos de morte, realização de necropsias com colheita de material para estudo histopatológico. Os dados epidemiológicos registrados foram: localização da propriedade; espécies de Brachiaria ou de outros gêneros presentes no piquete onde os animais pastavam; número e espécies de animais na propriedade; idade e sexo dos animais; idade ao desmame; procedência dos animais; tempo de permanência dos animais na pastagem; tipo de pastejo; época em que ocorria a doença (seca ou chuvosa); área da pastagem; tempo de implantação da pastagem; estágio do capim; realização de adubação; ocorrência de fotossensibilização em épocas anteriores e condições que ocorreram as mesmas; frequência da enfermidade: morbidade e letalidade. O exame clínico geral dos animais foi realizado segundo as recomendações de Diffay (2004). Foram colhidas amostras de sangue para determinação dos níveis séricos de gama glutamiltransferase (GGT), aspartato aminotranferase (AST), bilirrubina direta (BD), bilirrubina indireta $(\mathrm{BI})$, bilirrubina total $(\mathrm{BT})$, ureia e creatinina, usando kits colorimétricos através de espectrofotômetro, no laboratório de Patologia Clínica da Central de Diagnóstico Veterinário (CEDIVET) da Universidade Federal do Pará (UFPA), Castanhal-PA. Os animais que apresentavam sinais clínicos de fotossensibilização foram retirados das pastagens para evitar mortes. Em caso de morte, foi realizada necropsia e colhidos fragmentos de diversos órgãos, os quais foram acondicionados em formol a $10 \%$. O material coletado durante a necropsia foi enviado para realização de exame histológico, sendo processado rotineiramente e corado pela Hematoxilina-Eosina (HE) no Setor de Anatomia Patológica Veterinária da CEDIVET/UFPA e no Setor de Anatomia Patológica do Convênio "Projeto Sanidade Animal Embrapa/Universidade Federal Rural do Rio de Janeiro". Foram colhidas amostras de $B$. brizantha, antes da introdução dos animais no experimento e após a apresentação de sinais clínicos dos mesmos, bem como na ocasião dos surtos, para a determinação do teor de saponinas qualitativamente, pelo método de cromatografia de camada delgada (Gjulemetowa et al. 1982) e quantitativamente por HPLC utilizando o detector de evaporação por dispersão de luz (Evaporative Light Scattering Detector) (Wang et al. 2004) Estas análises foram realizadas no Laboratório de Química do Instituto Biológico de São Paulo. No momento da colheita de amostras do capim para dosagem do teor de saponinas, também foram colhidas amostras para contagem de esporos de $P$. chartarum, realizada através do "wash method" de acordo com Menna (1973). Para o estudo de morbidade foi utilizado o teste Qui-quadrado com o intuito de verificar se as distribuições dos animais doentes, do grupo que receberam suco de rúmen e do grupo que não receberam suco de rúmen, eram semelhantes. Para o estudo de letalidade foram avaliados somente os animais doentes e utilizado o teste exato de Fisher. Para os testes foi considerado o nível crítico de probabilidade de $5 \%(p<0,05)$ (Callegari-Jaques 2003). A análise de variância foi feita com base no teste $\mathrm{F}$ e para a comparação de médias foi usado o teste de Tukey, todos ao nível de significância de $5 \%(p<0,05)$. Para essas análises foi usado o programa NTIA versão 4.2.1 de outubro de 1995, desenvolvido pela EMBRAPA de Campinas.

\section{Surto 1}

\section{RESULTADOS}

Em uma fazenda localizada no município de Santa Luzia do Pará, no mês de outubro de 2008, correspondente a época seca na região, foi diagnosticado um surto fotossensibilização em um rebanho de 113 cordeiros, cruzas de ovelhas Santa Inês com carneiros Dorper, de ambos os sexos, com idade entre dois e três meses. Esses cordeiros nasceram na própria fazenda e foram mantidos até um a dois meses de idade em apriscos suspensos, recebendo capim-elefante roxo (Pennisetum purpureum cv. roxo), concentrado comercial, sal mineral e água ad libitum. Após esse período, foram introduzidos na pastagem de Brachiaria brizantha que se encontrava com massa residual reduzida e senescente (Fig.1). Segundo o tratador, após quinze dias de pastejo, durante oito horas por dia, os animais começaram a apresentar sinais clínicos. Dos 113 cordeiros, adoeceram 49 e morreram 40 . As taxas de morbidade e letalidade nesse surto foram de $43,4 \%$ e $81,6 \%$, respectivamente. A morte dos animais ocorreu, em média, após

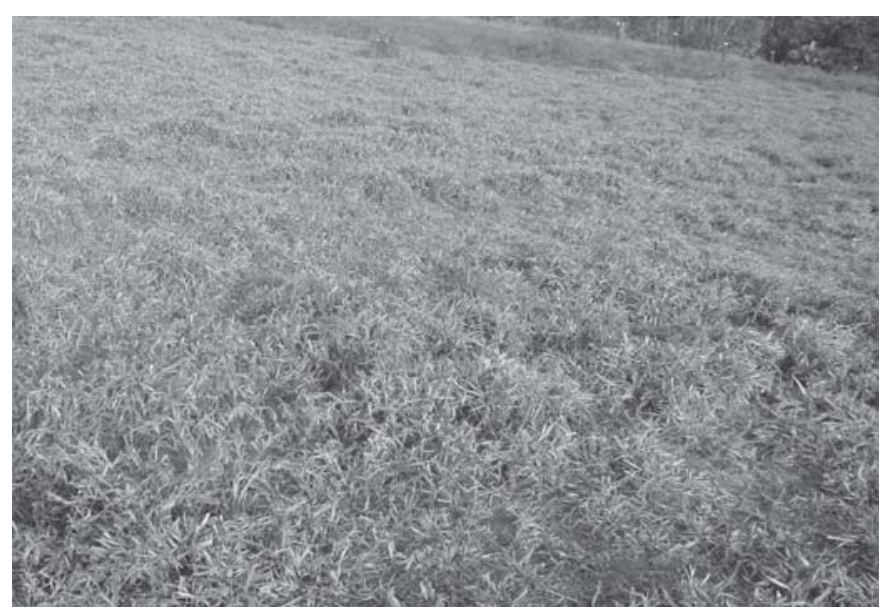

Fig.1. Pastagem de capim Brachiaria brizantha, com massa residual reduzida e senescente, na qual foram introduzidos os ovinos do Surto 1. 
duas semanas do início dos sinais clínicos. Nessa propriedade, a pastagem havia sido implantada há mais de quinze anos e nunca havia sido realizada adubação. No entanto, a criação de ovinos só foi iniciada seis anos após a implantação da pastagem. Os tratadores não sabiam informar se haviam ocorrido surtos em épocas anteriores.

\section{Surto 2}

Ocorreu na mesma fazenda do Surto 1 , no mês de janeiro de 2009, época de início das chuvas, porém em outro piquete de capim $B$. brizantha, em um lote de 80 cordeiros, cruza de ovelhas Santa Inês x carneiros Dorper, nascidos na própria fazenda. Sob orientação técnica, os cordeiros foram introduzidos na pastagem após 15 dias de nascidos. Até então, eram mantidos sob o mesmo manejo dos animais do Surto 1. Após quinze dias de pastejo, durante oito horas por dia, os animais começaram a apresentar sinais clínicos. Dos 80 cordeiros, 13 adoeceram e 10 morreram. As taxas de morbidade e letalidade nesse surto foram $16,3 \%$ e $76,9 \%$, respectivamente. A morte dos animais ocorreu, em média, após duas semanas do início dos sinais clínicos.

\section{Experimento}

Sete dias após a introdução dos 49 cordeiros na pastagem, três dos que não haviam recebido fluido ruminal apresentaram sinais clínicos de fotossensibilização. No decorrer do experimento, dos 39 animais que não receberam fluido ruminal, $31(79,5 \%)$ adoeceram. Desses, 28 (90,3\%) morreram e três $(9,7 \%)$ se recuperaram. A média de evolução clínica desses animais foi de aproximadamente 11 dias. Dos 10 animais que receberam fluido ruminal, três $(30 \%)$ adoeceram, desses, todos morreram (100\%). A evolução clínica média nesses animais foi de aproximadamente 13 dias. Os índices de morbidade dos animais que receberam fluido ruminal são significativamente inferiores aos observados nos animais que não receberam o fluido, tendo em vista o teste qui quadrado $\chi^{2}(p<0,000001)$. Os índices de letalidade dos animais que não receberam o fluído ruminal, quando comparado aos animais que receberam, não têm diferença estatística significativa, tendo em vista o teste exato de Fisher $(p<0,00001)$.

\section{Sinais clínicos}

Os sinais clínicos apresentados tanto pelos animais dos surtos quanto pelos animais do experimento consistiam, inicialmente, em prurido, inquietação e fotofobia. Algumas vezes os cordeiros eram encontrados dentro de bueiros (Fig.2), embaixo dos apriscos e na encosta de árvores à procura de sombra. Após apresentavam edemas nas orelhas (Fig.3A), pálpebras, base da cauda (Fig.3B) e prepúcio; também apresentavam mucosas amareladas, com corrimento ocular (Fig.4) e cegueira. Na fase final, apresentavam lesões cutâneas que inicialmente se caracterizavam por dermatite acentuada (Fig.5) e, posteriormente, formação de crostas na região dos olhos, focinho, orelhas, dorso e membros. Alguns animais ainda apresentavam

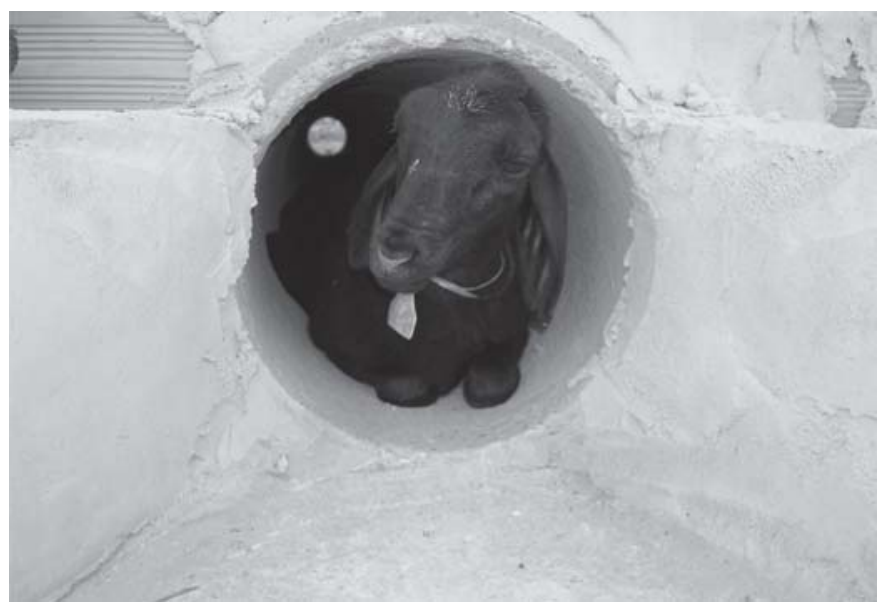

Fig.2. Cordeiro intoxicado por Brachiaria brizantha dentro de um bueiro na tentativa de se proteger dos raios solares.

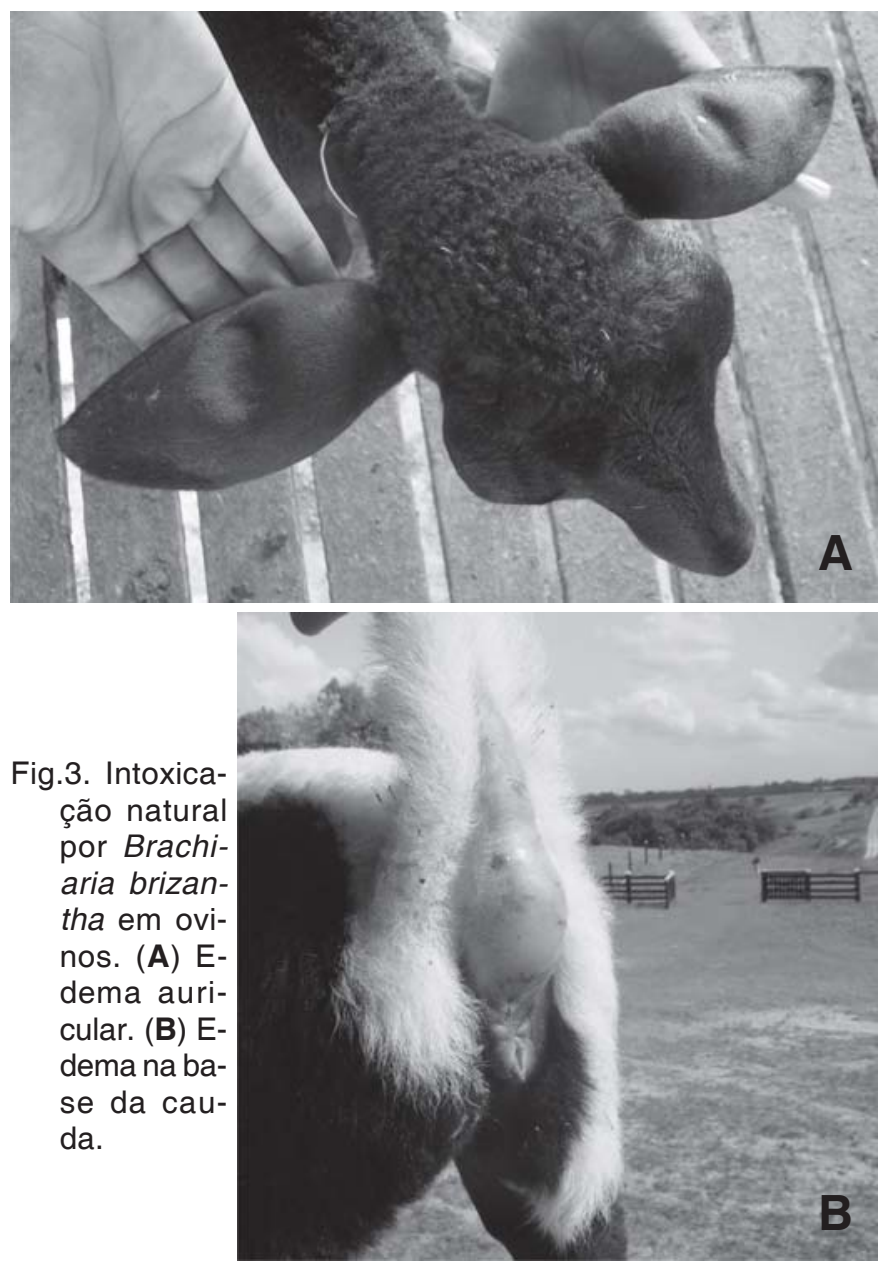

cólica, depressão, apatia, anorexia, além de sinais clínicos nervosos como incoordenação motora, tremor da cabeça, letargia, ataxia, depressão e coma antes da morte.

\section{Bioquímica hepática e renal}

Os achados bioquímicos das funções hepática e renal nos animais dos surtos encontram-se no Quadro 1. Onde 


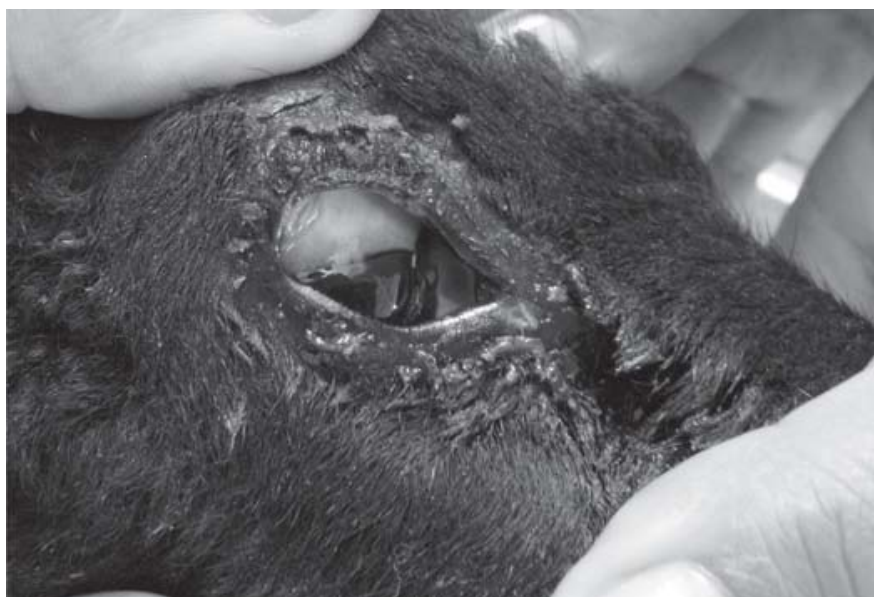

Fig.4. Esclera amarela, corrimento e retração do globo ocular em um ovino intoxicado naturalmente por Brachiaria brizantha.

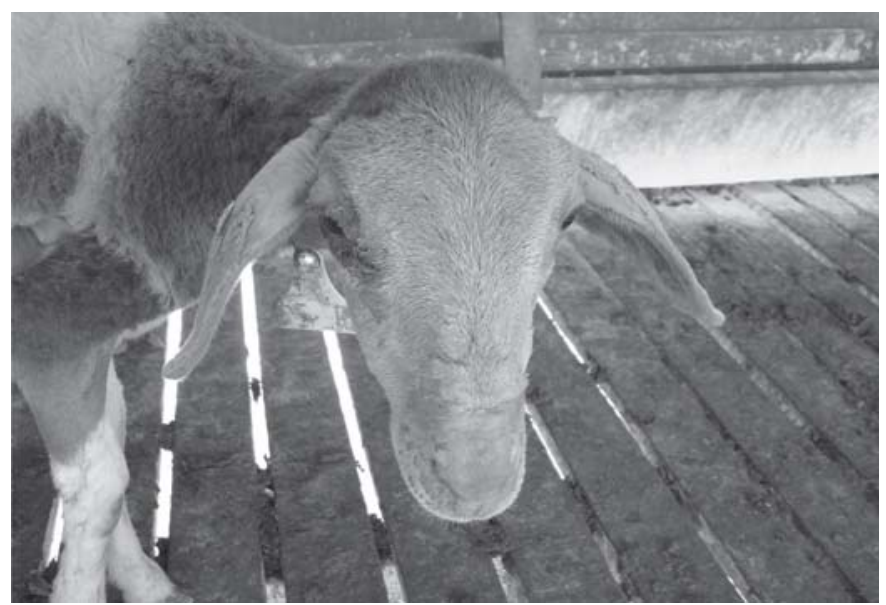

Fig.5. Dermatite acentuada em cordeiro na intoxicação natural por Brachiaria brizantha.

Quadro 1. Valores bioquímicos das funções renal e hepática em ovinos de dois surtos espontâneos de intoxicação por Brachiaria brizantha

\begin{tabular}{|c|c|c|c|c|c|c|}
\hline \multirow[t]{2}{*}{ Variáves } & \multirow{2}{*}{$\begin{array}{l}\text { Valores de } \\
\text { referência }\end{array}$} & \multirow[t]{2}{*}{ Unidade } & \multicolumn{2}{|c|}{ Surto 1} & \multicolumn{2}{|c|}{ Surto 2} \\
\hline & & & Med $^{b}$ & $\mathrm{DP}^{\mathrm{c}}$ & Med & DP \\
\hline & $17-43$ & & 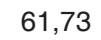 & I & 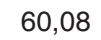 & 14,13 \\
\hline & & & & & & 5 \\
\hline & & & $38 \varepsilon$ & 20 & 378,14 & 198,45 \\
\hline $\mathrm{GC}$ & 0 & & 89,12 & 68,12 & 90,23 & 65,12 \\
\hline $\mathrm{BL}$ & 0 & $\mathrm{~g} / \mathrm{dL}$ & 1, & 1,78 & 168 & 175 \\
\hline $\mathrm{Bl}^{\mathrm{g}}$ & 0 & & 1,38 & 1,6 & 1,36 & 1,49 \\
\hline BT & $0,1-C$ & $\mathrm{ng} / \mathrm{dL}$ & 3,03 & 3,02 & 3,04 & 3,01 \\
\hline
\end{tabular}

a Kaneko et al.(1997); ${ }^{\text {média; }}$ ' desvio padrão; daspartato aminotransferase; ${ }^{\mathrm{e}} \gamma$-glutamil transferase; ${ }^{\mathrm{f}}$ bilirrubiona direta; ${ }^{9}$ bilirrubina indireta; ${ }^{\mathrm{h}}$ bilirrubina total.

se pode notar que os valores de ureia, creatinina, AST, GGT, BD, BI e BT apresentam aumento, quando comparados com os valores de referência. Os achados bioquímicos dos animais do experimento foram menores nos animais que receberam o fluido ruminal em comparação aos que não receberam $(p<0,0001)$. No entanto, os valores de ureia e GGT foram estatisticamente semelhantes para ambos os grupos, já os valores de creatinina, AST, BD, $B I$, e BT foram estatisticamente menores $(p<0,0001)$ nos animais que receberam fluido ruminal, em comparação aos que não receberam. Porém essa diferença nos valores de $\mathrm{BD}, \mathrm{BI}, \mathrm{e} \mathrm{BT}$ só foi verificada a partir da segunda semana (Fig. 6).

\section{Patologia}

As alterações encontradas na necropsia caracterizaramse por icterícia generalizada, edema de aspecto gelatinoso no tecido subcutâneo; o fígado apresentava coloração amarelada, consistência aumentada e padrão lobular acentuado. A vesícula biliar encontrava-se distendida, com bile escura e espessa. Nos rins foi observada coloração amareloesverdeado e aumento de tamanho. As alterações histológicas ocorreram principalmente no fígado e podem ser descritas como leve proliferação das vias biliares nos espaços porta, proliferação de macrófagos, leve megalocitose de hepatócitos, presença de hepatócitos binucleados, presença de macrófagos espumosos, necrose de hepatócitos isolados (Fig. 7), colangite e presença de cristais em macrófagos e hepatócitos (Fig. 8). Nos rins foram observadas leves alterações degenerativas das células epiteliais dos túbulos uriníferos, sob a forma de tumefação.

\section{Determinação de saponinas}

Foram determinados dois tipos de saponinas nas amostras de $B$. brizantha dos surtos e do experimento, a metilprotodioscina e a protodioscina. Os níveis de saponina em B. brizantha no Surto 1 e no Surto 2 foram 0,92\% e 0,88\%, respectivamente. No experimento, os níveis variaram de $1,13 \%$ a $1,62 \%$.

\section{Contagem de esporos de Pithomyces chartarum}

Em cada surto, a contagem de esporos de $P$. chartarum, revelou a presença de dois esporos por grama de folhas secas; no experimento foi observada a presença de um esporo, antes da introdução dos animais na pastagem e após a ocorrência de sinais clínicos.

\section{DISCUSSÃO E CONCLUSÃO}

O diagnóstico de fotossensibilização associada à ingestão de capim Brachiaria brizantha baseou-se na epidemiologia, sinais clínicos, achados bioquímicos e histopatológicos, bem como, pela quantidade insignificante do fungo Pithomyces chartarum e pela presença de saponinas na pastagem, como descrito por outros autores (Salam Abdullah et al. 1988, Lemos et al. 1996a). Foi verificado que os animais jovens, dos surtos, foram mais sensíveis à intoxicação pela $B$. brizantha do que os adultos, visto que somente os cordeiros adoeceram. Os cordeiros do experimento também foram muito sensíveis à intoxicação, apresentando sinais clínicos severos, como os observados por Lemos et al. (1996a). As altas taxas de morbidade e letalidade observadas nos cordeiros que não receberam fluido ruminal, também foram observadas no Surto 1. Isso 

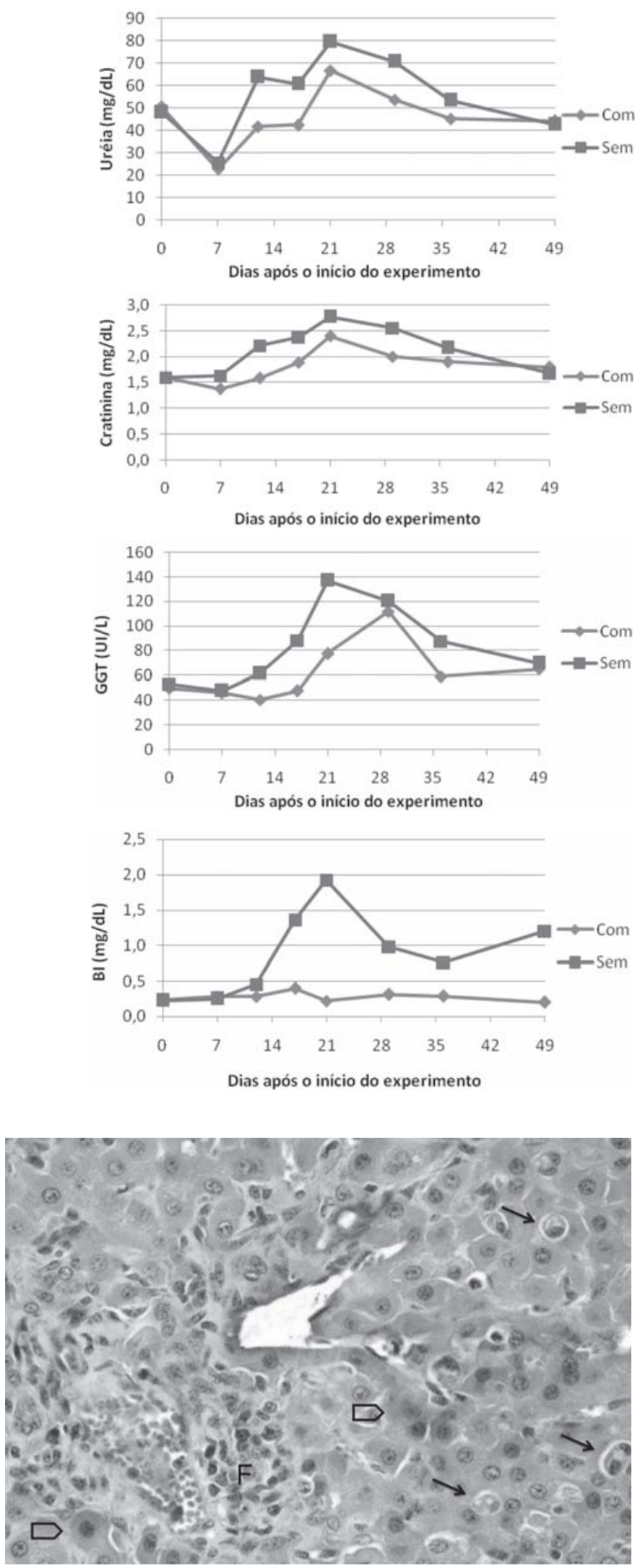

Fig.7. Espaço porta com discreta fibrose (F), necrose individual de hepatócitos (cabeça de seta) e macrófagos espumosos em sinusoides (seta). HE, obj.20x.
Fig.6. Valores médios de ureia, creatinina, aspartato aminotransferase (AST), gama glutamiltransferase (GGT), bilirrubina direta (BD), bilirrubina indireta $(\mathrm{BI})$ e bilirrubina total $(\mathrm{BT})$, ao longo do período do experimento, dos animais que receberam o fluido ruminal (Com) e dos animais que não receberam (Sem).
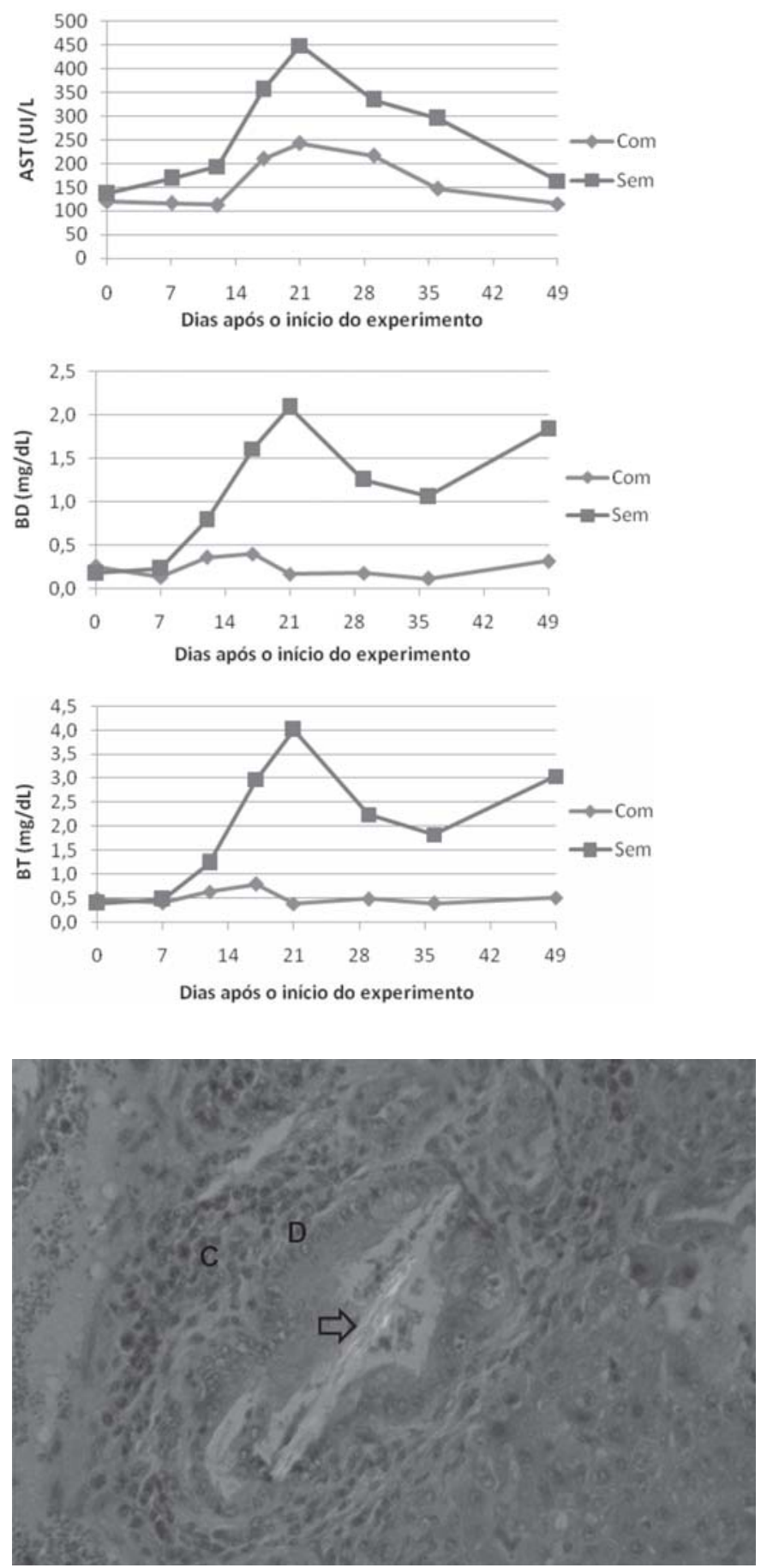

Fig.8. Ducto biliar (D) envolto por infiltrado inflamatório mononuclear; (C) no lúmen do ducto há cristal refringente sob luz polarizada (seta). HE, obj.40x. 
pode ter ocorrido em função dos animais terem sido mantidos nos primeiros 60 dias de vida em apriscos e posteriormente introduzidos na pastagem de $B$. brizantha, o que provavelmente não possibilitou uma adaptação prévia, visto que a taxa de morbidade diminuiu consideravelmente no Surto 2, após ser recomendado ao proprietário a introdução dos animais na pastagem poucos dias após o nascimento. De acordo com Brum et al. (2004) animais introduzidos pela primeira vez em pastagem de Brachiaria spp. são mais suscetíveis a intoxicação. As saponinas determinadas nas amostras de $B$. brizantha neste estudo, confirmam o que já foi observado em diversas regiões do Brasil, que as pastagens de Brachiaria spp. contêm saponinas esteroidais litogênicas e são responsáveis por fotossensibilização hepatógena (Souza et al. 2006, Brum et al. 2009). Foi verificada maior quantidade de saponina no capim B. brizantha no período seco, o que está de acordo com o observado por Souza et al. (2006), e com o capim em fase de maturação, como demonstrado por Brum et al. (2009). Nessa época o capim se encontrava com poucos centímetros de altura e o pastejo era intensivo, diferente do observado por Brum (2004), que verificaram que nessas condições o capim é menos tóxico para ovinos. A quantidade de saponina pode variar na mesma espécie de pastagem devido a vários fatores, tais como o estresse ambiental, idade da planta e a fase de desenvolvimento (Oleszek 2002). Isso, de certa forma, pode explicar a variação na quantidade saponina observada no decorrer do experimento. A diminuição na taxa de morbidade, estatisticamente significativa $(p<0,0001)$, e os menores valores observados na bioquímica dos animais que receberam fluido ruminal, em comparação aos animais que não receberam, podem ser justificados pela administração do fluido ruminal das mães que provavelmente já estavam adaptadas aos compostos tóxicos presentes no capim, já que as mesmas não adoeceram; esse fluido pode ter induzido à mudança na população microbiana do rúmen dos cordeiros, auxiliando na degradação de compostos tóxicos presentes no capim, que os mesmos pastavam. Estando de acordo com Dawson \& Allison (1988), os quais relatam que a mudança na população microbiana do rúmen pode ser considerada como um meio de adaptação. Os achados clínicos e patológicos apresentados pelos ovinos intoxicados por capim B. brizantha, nesse estudo, foram semelhantes aos encontrados na intoxicação por outras espécies de Brachiaria descrita por outros autores (Lemos et al. 1996a, Driemeier et al. 1998, Cruz et al. 2001). Um aspecto digno de nota foram os sinais clínicos neurológicos apresentados por alguns dos animais intoxicados, também observados por Lemos et al. (1996a) e Cruz et al. (2001). Isso pode ter ocorrido porque, normalmente, substâncias tóxicas como a amônia, ácidos graxos de cadeias curtas e mercaptanos são eliminadas quando passam pelo fígado, o que não ocorre quando há lesão hepática difusa grave com insuficiência hepática; em consequência essas substâncias podem chegar ao encéfalo e como falsos neurotransmissores causar vários sinais clínicos neurológicos
(Kelly 2002). Um achado importante na histologia do fígado dos ovinos intoxicados por $B$. brizantha, foi a presença de material cristaloide no sistema biliar e colangite. Segundo Miles et al. (1991) e Cruz et al. (2001), esses achados associados a sinais clínicos de fotossensibilização, indicam a presença de saponinas esteroidais na pastagem, o que foi confirmado através da pesquisa dessas substâncias na pastagem de $B$. brizantha dos animais do experimento e dos surtos.

Agradecimentos.- À Coordenação de Aperfeiçoamento de Pessoal de Nível Superior (CAPES), pela concessão de bolsa de mestrado. Ao proprietário da fazenda estudada, pela contribuição na realização deste trabalho. À Dra Mitsue Haraguchi, pela determinação de saponinas. Ao Dr. David Driemeier da UFRGS, pela valiosa ajuda neste trabalho. Ao projeto Milenium, coordenado pelo Dr. Franklin Riet Correa, pelo apoio financeiro.

\section{REFERÊNCIAS}

Albernaz T.T., Silveira J.A.S., Reis A.B., Oliveira C.H.S., Oliveira C.M.C., Duarte M.D., Cerqueira V.D., Riet-Correa G. \& Barbosa J.D. 2008. Fotossensibilização em ovinos associada à ingestão de Brachiaria brizantha no Pará. Anais do Encontro Nacional de Diagnóstico Veterinário, Campo Grande, MS, p.73-74.

Barbosa J.D., Oliveira C.M.C., Torkarnia C.H. \& Peixoto P.V. 2006. Fotossensibilização hepatógena em equinos pela ingestão de Brachiaria humidicola (Gramineae) no Estado do Pará. Pesq. Vet. Bras. 26:147-153.

Brum, K.B. 2006. Papel das saponinas e do Pithomyces chartarum como agentes hepatotóxicos para ruminantes em sistemas de pastejo. Dissertação de Mestrado em Medicina Veterinária, Escola de Veterinária da Universidade Federal de Goiás (Goiânia), GO. 93p.

Brum K.B., Haraguchi M., Garutti M.B., Nóbrega F.N., Rosa B. \& Fioravante M.C. 2004. Análise semiquantitativa da saponina protodioscina do ciclo vegetativo de Brachiaria decumbens. Pesq. Vet. Bras. 24(Supl.):13-14.

Brum K.B., Haraguchi M., Garutti M.B., Nóbrega F.N., Rosa B. \& Fioravante M.C. 2009. Steroidal saponin concentrations in Brachiaria decumbens and $B$. brizantha at different developmental stages. Ciência Rural 39:279-281.

Callegari-Jacque S.D. 2003. Bioestatística: princípios e aplicações. Artmed, Porto Alegre. 255p.

Cruz C., Driemeier D., Pires V.S. \& Schenkel E.P. 2001. Experimentally induced cholangiohepatopathy by dosing sheep with fractionated extracts from Brachiaria decumbens. J. Vet. Diagn. Invest.13:170172.

Dawson K.A. \& Allison M.J. 1988. Digestive Disorders and nutritional toxicity, p.445-459. In: Hobson P.N. (Ed.), The Rumen Microbial Ecosystem. Elsevier Applied Science, New York. 826p.

Diffay B.C., Mckenzie D., Wolf C. \& Pugh D.G. 2004. Abordagem e exame de ovinos e caprinos, p.1-19. In: Pugh D.G. (Ed.), Clínica de Ovinos e Caprinos. Roca, São Paulo. 528p.

Driemeier D., Barros S.S., Peixoto P.V., Tokarnia C.H., Döbereiner J. \& Brito M.F. 1998. Estudo histológico, histoquímico e ultra-estrutural de fígados e linfonodos de bovinos com presença de macrófagos espumosos ("foam cells"). Pesq. Vet. Bras. 18:29-34.

Driemeier D., Colodel E.M., Seitz A.L., Barros S.S. \& Cruz C. 2002. Study of experimentally induced lesions in sheep by grazing Brachiaria decumbens. Toxicon 40:1027-1031.

Döbereiner J., Tokarnia C.H., Monteiro M.C.C., Cruz L.C.H., Carvalho E.G. \& Primo A.T. 1976. Intoxicação de bovinos e ovinos em pastos de Brachiaria decumbens contaminados por Pithomyces chartarum. Pesq. Agropec. Bras., Sér. Vet. 11:87-94. 
Fioravante M.C. 1999. Incidência, avaliação clínica, laboratorial e anatomopatológica da intoxicação subclínica por esporidesmina em bovinos. Tese de Doutorado em Medicina Veterinária, Faculdade de Medicina Veterinária e Zootecnia, Unesp, Botucatu, SP. 93p.

Gjulemetowa R., Tomowa M., Simowa M., Pangarowa T. \& Peewa S. 1982. Über die Bestimmung von Furostanolsaponinen im Präparat Tribestan ${ }^{\circledR}$. Pharmazie 37:296.

IBGE 2008. Coordenadas Geográficas. Instituto Brasileiro de Geografia e Estatística. Disponível em <http:// www.ibge.gov.br> Acesso em 15 jan. 2009.

Kaneko J.J., Harvey J.W. \& Bruss M.L. 1997. Clinical biochemistry of domestic animals, p. 890-894 5th ed. Academic Press, New York.

Kelly W.R. 2002. Enfermedad del hígado en grandes y pequeños rumiantes. Anais $10^{\circ}$ Congresso Latinoamericano de Buiatria, Paysandú, Uruguai, p.1-6.

Lemos R.A.A., Ferreira L.C.L., Silva S.M., Nakazato L. \& Salvador S.C. 1996a Fotossensibilização e colangiopatia associada a cristais em ovinos em pastagem com Brachiaria decumbens. Ciência Rural 26:109-113.

Lemos R.A.A., Ferreira L.C.L., Salvador S.C. \& Nakazato L. 1996b. Fotossensibilização e colangiopatia associada a cristais em bovinos mantidos em pastagens de Brachiaria decumbens no Mato Grosso do Sul. Anais 1ำ Encontro de Laboratórios de Diagnóstico Veterinário do Cone Sul, Campo Grande, MS, p.41-43.

Lemos R.A.A., Salvador S.C. \& Nakazato L. 1997. Photosensitization and crystal associated cholangiohepatopathy in cattle grazing Brachiaria decumbens in Brazil. Vet. Hum. Toxicol. 39:376-377.

Lemos R.A.A., Nakazato L., Herrero Jr G.O., Silveira A.C. \& Porfirio L.C. 1998. Fotossensibilização e colangiopatia associada a cristais em caprinos mantidos sob pastagens de Brachiaria decumbens no Mato Grosso do Sul. Ciência Rural 28:507-510.
Menna M.E. 1973. Facial Eczema, 2. Warning systems. Proc. Ruakura Farmer's Conference. New Zealand, p.50-54.

Miles C.O., Munday S.C., Holland P.T., Smith B.L., Embling P.P. \& Wilkins A.L. 1991. Identification of a sapogenin glucoronide in the bile of sheep affected by Panicum dichotomiflorum toxicosis. N. Z. Vet. J. 39:150-152.

Oleszek W.A. 2002. Chromatographic determination of plant saponins. J. Chromatography 967:147-162.

Resende R.M.S., Valle C.B. \& Jank L. 2008. Melhoramento de forrageiras tropicais. Embrapa Gado de Corte, Campo Grande, MS. 293p.

Rozza D.B., Seitz A.L., Bandarra P.M., Santos E.O. \& Driemeier D. 2004. Fotossensibilização por Brachiaria decumbens em búfalo. Pesq. Vet. Bras. 24(Supl.):55-56.

Salam Abdullah A., Nordin M.M. \& Rajion M.A. 1988. Signal grass (Brachiaria decumbens) toxicity in sheep: changes in motility and $\mathrm{pH}$ of reticulo-rumen. Vet Hum. Toxicol. 30:256-258.

Silveira J.A.S., Albernaz T.T., Silva N.S., Lopes C.T.A, Cerqueira V.D., Oliveira C.M.C., Duarte M.D. \& Barbosa J.D. 2009. Fotossensibilização hepatógena em caprinos associada à ingestão de Brachiaria brizantha no Estado do Pará. Anais 8ํㅡㄹ Congresso Brasileiro de Buiatria, Belo Horizonte, MG, p.336-341.

Smith B.L. \& Miles C.O. 1993. A role for Brachiaria decumbens photosensitization of ruminants. Vet. Hum. Toxicol. 35:256-257.

Souza V.S., Brum K.B., Garutti M. B., Fioravanti M.C.S. \& Haraguchi M. 2006. Influência da sazonalidade e pluviometria sobre a saponina esteroidal das gramíneas Brachiaria brizantha e Brachiaria decumbens em Jataí (GO). Anais Reunião Anual da Sociedade Brasileira de Química, Águas de Lindóia, SP, p.29.

Wang H., Provan G.J. \& Helliwell K. 2004. Determination of rosmaric acid and caffeic acid in aromatic herbs by HPLC. Food and Chemistry 87:307-311. 